\title{
La relación entre ética y política en la democracia deliberativa. Un análisis crítico de la "Significación moral de la democracia", de Julio De Zan
}

\section{The relationship between ethics and politics in deliberative democracy. A critical analysis of "Significación moral de la democracia", by Julio De Zan}

Santiago Prono*

\begin{abstract}
Resumen: El artículo analiza el problema de la relación entre ética y política teniendo en cuenta para ello el trabajo de J. De Zan sobre la "Significación moral de la democracia" (1991). La idea es complementar, no sin señalamientos críticos, dicho trabajo identificando, en primer lugar, el sentido reconstructivo del planteamiento teórico de la democracia deliberativa de J. Habermas. Para este propósito, se explicita también una parte de sus fundamentos filosóficos, como es la pragmática universal del lenguaje, cuya tematización de las pretensiones universales de validez del discurso argumentativo permite identificar el carácter moralmente constitutivo de la racionalidad argumentativa, y por lo tanto de la democracia deliberativa. Aunque de un modo indirecto, este trabajo se propone discutir críticamente también con el actual posicionamiento habermasiano sobre el tema.
\end{abstract}

Palabras clave: De Zan, democracia deliberativa, política, moral, sentido reconstructivo.

Abstract: The article analyzes the problem of the relationship between ethics and politics taking into account the work of J. De Zan "Significación moral de la democracia" (1991). The idea is to complement, not without critical observations, this

\footnotetext{
* Doctor en Filosofía por la Universidad de Buenos Aires, Investigador Adjunto del CONICET y Profesor Adjunto en la Universidad Nacional del Litoral. Es director de proyectos de investigación de esta universidad y del CONICET. Ha sido becario del DAAD para realizar estancias de investigación en la Freie Universität Berlin, y en la Goethe Universität Frankfurt. Sus principales líneas de trabajo se relacionan con la teoría del discurso de J. Habermas y de K.-O. Apel, tema sobre el que ha publicado libros y artículos en revistas especializadas. Dirección electrónica: santiagoprono@hotmail.com
} 
work identifying, in the first place, the reconstructive sense of the theoretical approach to the deliberative democracy of J. Habermas. For this purpose, a part of its philosophical foundations is also made explicit, such as the universal pragmatics of language, whose thematization of the universal claims of validity of argumentative discourse makes it possible to identify the morally constitutive character of argumentative rationality, and therefore of the deliberative democracy. Although indirectly, this paper proposes to discuss critically also with the current Habermasian position on the subject.

Keywords: De Zan, Deliberative Democracy, Politics, Moral, Reconstructive Sense.

\section{Introducción}

Ya sea de manera explícita o implícita, desde la Antigüedad clásica se analiza la relación entre ética y política. Por supuesto, se trata de un análisis que, a lo largo de los siglos y años precedentes, distintos autores y corrientes de pensamiento han venido realizando desde diversas (y divergentes) perspectivas teóricas. Desde Platón, para quien el régimen político debía organizarse de modo que sea capaz de expresar la idea de Bien, pasando por los filósofos medievales y la consecuente necesidad de someter el orden político terrenal a los designios de su Dios celestial, o en los albores de la Modernidad, cuando los filósofos se encontraban "poco convencidos de la existencia" divina, ${ }^{1}$ el tema de la mencionada relación no ha podido definirse de manera de alcanzar un consenso (ni siquiera provisorio o general). En la actualidad, y aunque por cierto partiendo de diferentes marcos o "paradigmas" conceptuales, algunos análisis teóricos de la política se realizan desde el punto de vista moral pretendiendo fundamentar, por ejemplo, el proyecto de una ética mundial como condición de posibilidad de la supervivencia a nivel global, y por supuesto de todo ordenamiento socio-político. ${ }^{2}$ Más recientemente, también se han analizado los problemas político-morales de las sociedades plurales contemporáneas desde una perspectiva ética, teniendo en cuenta su aspecto educacional como medio para promover los razonamientos prácticos

${ }^{1}$ Cfr. Belaval, I. (comp.), Historia de la filosofia, Vol. 6: Racionalismo, empirismo, Ilustración, México, Siglo XXI, 1998, p. 200; véase también pp. 1-6, 195-203.

${ }^{2}$ Cfr. Küng, H., Proyecto de una ética mundial, Madrid, Trotta, 2006. 
necesarios, y ello a fin de mejorar las habilidades personales en el marco de un ordenamiento político democrático. ${ }^{3}$ Ahora bien, en este marco el presente artículo analiza críticamente un trabajo de Julio De Zan en el que expuso su concepción respecto de la "significación moral de la democracia"4. En este sentido, se sostiene que tal planteo respecto de la relación allí analizada entre política y moral se justifica teniendo en cuenta los fundamentos filosóficos de la teoría del discurso (presupuesta por la democracia deliberativa de J. Habermas). ${ }^{5}$ El valor de tal aproximación filosófica al tema se basa en que el mismo no ha sido convenientemente considerado por aquel autor, lo cual evidencia cierta carencia conceptual en la señalada significación. La idea entonces, con este análisis, es dotar del correspondiente fundamento teórico a la idea de que en efecto es conceptualmente posible identificar una conexión entre ética y política en el marco teórico de la democracia deliberativa.

El siguiente es el plan de trabajo propuesto para el mencionado análisis desde el punto de vista señalado. Luego de identificar algunos de los principales planteos filosóficos contemporáneos que analizan el tema de la relación entre política y moral desde el marco teórico de la ética del discurso, se presenta y analiza críticamente el planteo de Zan (en el citado trabajo de 1991) como propuesta para identificar la relación entre ética y política (1). A fin de dotar de

3 Serodio, A.; Kopelman, B., Bataglia, P., "Promoting moral and democratic competencies: towards an educational turn of Bioethics". En Revista Bioética, 24, 2, Brasilia, May/Aug 2016, pp. 235-242.

${ }^{4}$ Trabajo publicado con dicho título en Apel, K.-O., Cortina, A., De Zan, J., Michelini, D. (eds.), Ética comunicativa y democracia, Barcelona, Crítica, 1991, pp. 297-320.

${ }^{5}$ Los términos "ética" y "moral" aquí utilizados, refieren a dos puntos de vista interrelacionados, concibiendo a esta en el sentido de la forma incondicionada del deber y la obligación con pretensiones de validez universal, y a la ética como el conjunto de creencias, actitudes o ideales que configuran una "personalidad cultural básica", pero sin negar la posibilidad de fundamentación racional. Un análisis de este tema está en Prono, S., Democracia deliberativa y Estado democrático de derecho. Análisis y contribuciones desde la teoría del discurso, Santa Fe, Ediciones UNL, 2018, p. 109 y ss.; 2014, "Ética del discurso y democracia deliberativa. Un análisis desde la pragmática trascendental del lenguaje de K.-O. Apel", en Erasmus. Revista para el diálogo intercultural, XVI, 1, pp. 2541; 2010, "Ética y política. Reflexiones críticas en torno a la relación entre la ética del discurso y la democracia deliberativa"; Revista de Filosofia, Universidad Industrial de Santander (Bucaramanga, Colombia), 9, 2, 2010, pp. 59-86. 
mayor respaldo teórico a tal identificación, se procede, primero, a una presentación general de la democracia deliberativa de J. Habermas (2), para después explicitar la relación entre política y moral en el marco de esta teoría política. Esto se realiza, en primer lugar, señalando algunas notas distintivas del sentido reconstructivo de la filosofía habermasiana (3), y ello a fin de explicitar parte del trasfondo filosófico presupuesto por aquella teoría política (4), relacionado con la pragmática universal del lenguaje y la consecuente identificación o descubrimiento de las pretensiones universales de validez del discurso argumentativo, sólo y parcialmente mencionado en el trabajo de Zan (4.a). La idea es que la tematización de tales pretensiones de validez permite explicitar el carácter moralmente inherente de la democracia deliberativa (4.b). Las reflexiones finales consisten en una breve formulación de algunas aclaraciones conceptuales respecto de la aproximación teórica al tema en la obra filosófica (previa y posterior) de Zan sobre temas de filosofía práctica (5).

\section{La relación entre política y moral en la teoría ética del discurso}

Desde el punto de vista de la teoría ética del discurso, K.-O. Apel ha considerado a la filosofía política como una parte de dicha teoría: a su entender, la filosofía política estriba en una aplicación de la ética del discurso en el sentido de su parte B, que analiza el problema de la fundamentación de la aplicación del principio básico (Grundprinzip), pragmático-trascendentalmente fundamentado, y su consecuente exigencia de resolver conflictos en base a consensos racionalmente motivados. Ahora bien, se trata ésta de una parte de la teoría por la cual, sostiene el autor, la misma tiene que concebirse como ética de la responsabilidad referida a la historia $\mathrm{y}$, en este sentido, también para la legitimación del sistema político a la luz de una idea regulativa a largo plazo. ${ }^{6} \mathrm{~A}$

${ }^{6}$ Cfr. Apel, Transformation der Pbilosophie (1973), Frankfurt, Suhrkamp, Tomo I, 1999, p. 427, "Diskursethik vor der Problematik von Recht und Politik: Können die Rationalitätsdifferenzen zwischen Moralität, Recht und Politik selbst noch durch die Diskursethik normativ-rational gerechtfertigt werden?", en Kettner, M., Apel, K.-O., Zur Anwendung der Diskursetbik in Politik, Recht und Wissenschaft, Frankfurt, Suhrkamp, 1993, pp. 29-61, "Diskursethik als Ethik der Mit-Verantwortung vor den Sachzwängen der Politik, des Rechts und der Marktwirtschaft”, en Apel, K,.O, Burckhart, H., Prinzip 
su vez, una parte importante de sus discípulos que analizan la política lo hace (también) desde el punto de vista de dicha teoría ética, y ello de un modo que parece desconocer a la filosofía política el estatuto de una disciplina filosófica autónoma. En este sentido, D. Böhler ha señalado que el Estado democrático de derecho está condicionado a establecer discursos morales, pues ante la pregunta de “¿cómo puede la razón práctica concretarse políticamente?”, la respuesta dada es que ello es posible "si se considera que la fundamentación discursiva de las normas se orienta tanto a las normas morales últimas como así también al procedimiento democrático", y que para esto es necesaria una "orientación universalista y moral": de otro modo tal procedimiento de fundamentación sería moralmente ciego e irracional, anulando toda posibilidad de legitimidad política.7 Por esto sostiene también W. Kuhlmann que "las instituciones políticas, que deben intervenir profunda y eficazmente en las relaciones existenciales de los individuos por ellas afectados, deben ser fundadas y diseñadas conforme a los puntos de vista morales de modo que (tales instituciones) resulten aceptables para todos"8. Por su parte, M. Kettner también ha relacionado en este sentido la Filosofía política con la ética al identificar las actividades de las organizaciones no gubernamentales de la

vor den Mitverantwortung. Grundalge für Ethik und Pädagogik, Würzburg, Königshausen \& Neumann, 2001, p. 88.

${ }^{7}$ Böhler, D., Funkeolleg. Studientexte 3: Praktische Philosophie/Ethik, Weinheim und Basel, Beltz, 1984, pp. 868-869, y "Ethik und Zukunfts- und Lebenstverantwortung. Erster Teil: Begründung. Ziwischen Metaphysik und Reflexion im Dialog”, en Böhler, D., Brune, P., Orientierung und Verantwortung. Begegnungen und Ausenandersetzungen mit Hans Jonas, Würzburg, Könighausenn \& Neumanm, 2004, p. 151.

${ }^{8}$ Kuhlmann, W., "Ética do discurso e democracia”, en Amaro, F., Evânia, R., Jordan, M. (eds.), ¿Crisis da democracia?, Florianópolis, Nefiponline, 2015, p. 20. Otros comentaristas conciben a la teoría ética del discurso como desempeñando el rol de juez supremo de las prácticas políticas, porque resultaría "apropiada para actuar de árbitro en las sociedades democráticas a la hora de establecer o justificar normas" (Cortina A. "Ética discursiva y democracia política", en Revista colombiana de psicología, 2, 1993, p. 109), adoptando así un paternalismo orientado a educar al ciudadano a fin de que aprenda a colaborar y participar en la vida pública. Cfr. Camps, V., "Comunicación, democracia y conflicto”, en Apel, K.-O., Cortina, A., De Zan, J. y Michelini, D. (eds.), Ética comunicativa y democracia. Barcelona, Crítica, 1991, pp. 248, 249-250, 254; Kristol, I., "Moral and Ethical Development in a Democratic Society", en Moral Development. Princeton (NJ), Educational Testing Service, 1975, p. 175. 
sociedad civil (Nichtregierungsorganisationen) con el ejercicio de la política democrática, considerándolas como emergentes de la aplicación de la ética. En efecto, su tesis en este punto es que "en las democracias modernas las actividades de la ética aplicada son las actividades de la sociedad civil, y los actores de la ética aplicada tienen que plantear para sus actividades pretensiones que sean democráticamente válidas". ${ }^{9}$ Como puede observarse, también aquí se propone entonces una lectura ética de la política, en este caso, de las actividades que en tal sentido desarrollan las organizaciones de la sociedad civil en el contexto de la opinión pública política.

En el caso de Julio De Zan, el mismo ha pretendido fundamentar "la significación moral de la democracia" teniendo en cuenta parte de los presupuestos de la teoría ética del discurso, suscribiendo a la idea de que los fundamentos filosóficos de la democracia presuponen, y por ello se conectan con, la ética: de hecho alude a la viabilidad conceptual de una "fundamentación ética de la democracia" como punto de partida para su consolidación y ampliación. ${ }^{10}$ Así, en opinión del filósofo, es necesario contar con la posibilidad de formación de una conciencia moral esclarecida acerca de la validez moral del sistema democrático, cuyas convicciones deben fundarse, por supuesto, en argumentos racionales. ${ }^{11}$ Por esto señaló también que "los procedimientos tendientes a la formación de consenso, que resultan constitutivos de la democracia, se conectan esencialmente con principios morales y fines políticos que les transmiten su carácter valioso". ${ }^{12}$ Ahora bien, como puede ya apreciarse, este posicionamiento teórico de Zan acerca de la conexión entre política y moral pretende fundamentarse en el marco conceptual de la teoría del discurso: su tesis es que "(...) el principio del discurso constituye el criterio de legitimación de las decisiones colectivas y de las instituciones políticas, y posee

9 Kettner, M., "Welchen normativen Rahmen braucht die angewandte Ethik?", en Kettner, Matthias (ed.), Angewandte Ethik als Politikum, Frankfurt, Suhrkamp, 2000, p. 399.

${ }^{10}$ De Zan, "La significación moral de la democracia", p. 297.

${ }^{11}$ Ibidem, p. 301.

${ }^{12}$ Ibidem, p. 305. Subrayado nuestro. 
una relevancia moral fundamental y vinculante para la acción política en general". ${ }^{13}$

La pregunta que aquí se plantea es cómo procedió el autor para fundamentar esta tesis. La respuesta, aun cuando tenga en cuenta las implicancias conceptuales del discurso argumentativo, resulta sin embargo incompleta porque no parece haber profundizado en los fundamentos que señala. El trabajo de Zan se presenta así como una larga y teóricamente consistente conclusión del tema analizado, careciendo por momentos de una adecuada explicitación de los fundamentos que respaldan tales afirmaciones. En efecto, en numerosos pasajes del citado trabajo el mismo alude a las implicancias teóricas del principio del discurso, pero sin señalar ni por supuesto tematizar las pretensiones de validez que el mismo comporta, y en el marco de las cuales se expresa su valor moral, y por lo tanto también de la democracia.

De Zan suscribió a un concepto de democracia que explicita y reactualiza una idea que ha estado en el origen e inspirado su fundación, según la cual lo esencial de las democracias consistiría en la legitimación de las decisiones políticas por medio de consensos, y en la conexión de su validez con el discurso público, abierto a la participación igualitaria de todos los afectados (o sus representantes). ${ }^{14}$ Empero, a fin de justificar este carácter no-ideal ni abstracto de la moralidad inherente a una teoría discursiva de la democracia (i.e. teoría de la democracia deliberativa), corresponde explicitar una parte de sus fundamentos filosóficos, en este caso dados por la pragmática universal del lenguaje, que son también los fundamentos de la teoría ética del discurso. ${ }^{15}$

${ }_{13}^{13}$ Ibidem, p. 301.

${ }^{14}$ Cfr. De Zan, J., "La significación moral de la democracia", p. 305, La vieja y la nueva politica. Libertad, poder y discurso, Buenos Aires, UNSAM Edita, 2013, pp. 245 y ss.

15 Respecto de los fundamentos filosóficos de la democracia deliberativa, que por supuesto se vinculan con los (fundamentos) de la teoría del discurso de J. Habermas, además de la pragmática universal del lenguaje hay que mencionar también a la teoría de la acción social, en el marco de la cual se expresa el sentido originario de un uso consenso comunicativo (orientado al consenso) respecto de la interacción meramente estratégica del lenguaje (Habermas, 1981). Esta parte fundante de las pretensiones de validez de la política deliberativa, que permite responder a las aún persistentes objeciones basadas en la supuesta preeminencia del conflicto en las interacciones políticas, no es posible de analizar aquí. Posiciones que suscriben a tal concepción 
Precisamente esto es lo que falta en el análisis de Zan respecto del tema. La idea, entonces, es que procediendo a tal explicitación del marco teórico de la democracia deliberativa, que primero corresponde presentar en términos generales, es posible dotar de mayor respaldo conceptual a la justificación de la relación entre política y moral sugerida oportunamente por el filósofo, identificando así la mencionada "conexión esencial" entre los principios morales fundamentales y el ordenamiento político-democrático del Estado de derecho.

\section{Democracia deliberativa. Una introducción general}

La teoría de la democracia deliberativa presupone deliberación, intercambio de opiniones antitéticas que deben ser confrontadas en términos de argumentos como condición para tratar de obtener la mejor decisión posible respecto de un problema, y alcanzar así acuerdos racionalmente motivados. Esta teoría política, expuesta por Habermas en su principal obra filosófico-

conflictiva de la democracia, considerándola como esencialmente constitutiva de esta última, se encuentran, entre otros, en los estudios de Rancière, J., El desacuerdo. Política y filosofía, Buenos Aires, Nueva Visión, 1996; de Mouffe, Ch.; La paradoja democrática, Barcelona, Gedisa, 2003, y En torno a lo politico, Buenos Aires, FCE, 2007; de Touraine, A.; Crítica de la modernidad, Buenos Aires, FCE, 1994; o de Serrano Gómez, E.; Consenso y conflicto. Shmitt y Arendt: la definición de lo politico, Medellín, Editorial de la Universidad de Antioquia, 2002. Respuestas críticas a tales posicionamientos se encuentran en Maliandi, R., Ética: dilemas y convergencias, Buenos Aires, Biblos, 2006; en Michelini, D., "Ética discursiva y legitimidad democrática", en Apel K.-O., Cortina A., De Zan J., Michelini D. (eds.), Ética comunicativa y democracia, 2002, y Globalización, interculturalidady exclusión. Ensayos ético-politicos, Río Cuarto, ICALA; o en Prono, S., "Política y racionalidad discursiva. Algunas contribuciones para la reconstrucción de la democracia", en Cuadernos de Ética, 22, 35, 2007, pp. 83-61; "Democracia, conflicto y poder. Un abordaje conceptual desde la racionalidad discursiva", en Cuadernos filosóficos, V, Rosario, 2008, pp. 31-58; "Deliberative Demokratie. Einige Annäherungen an das Problem von Konsens vs. Konflikten", en Topologik. Rivista Internazionale di Scienze Filosofiche, Pedagogiche e Sociali. 6, Pellegrini, Cosenza, 2009, pp. 59-72, y "La democracia deliberativa y el problema de su implementación práctica. El debate consenso vs. conflicto", Universitas. Revista de filosofia, derecho y política, Universidad Carlos III (Madrid), 12, 2010, pp. 113-134. 
político-jurídica, Faktiz̨ität und Geltung (1992), ${ }^{16}$ asume un carácter normativo y procedimental (pero también sustantivo), cuyo principio básico no es el principio de la mayoría, sino el "principio del discurso", entendido como un cierto tipo de diálogo argumentativo esencial no sólo para conocer la realidad natural y social, sino también para evaluar las acciones y las instituciones humanas. Este principio, que desempeña un papel fundamental en esta teoría política y en las teorías del derecho y de la moral de Habermas (y también de Apel), presupone un procedimiento para los asuntos de validez o de justicia y en base al cual se legitiman las decisiones políticas: la idea es que estas decisiones tienen que adoptarse como resultado de una deliberación democrática, es decir, como un intercambio público de razones encaminadas a lograr el consenso. Habermas define este principio de la siguiente manera: "Sólo son válidas aquellas normas de acción a las que todas las personas posiblemente afectadas podrían dar su asentimiento como participantes en discursos racionales"17.

La idea contenida en este principio presupone una forma de diálogo (que en realidad podría caracterizarse como un "diálogo crítico") en cuyo contexto se busca el fundamento de las problemáticas pretensiones de validez de las opiniones y normas. Tal fundamento es necesariamente dialógico y exige sobre todo la simetría y la correspondiente igualdad de derechos entre quienes participan en el mencionado procedimiento. La simetría consiste en la pretensión normativa según la cual todos los posibles participantes tienen el mismo derecho de criticar y el mismo deber de justificar sus opiniones atendiendo exclusivamente a "la fuerza de coacción" que sólo los mejores argumentos pueden ejercer. ${ }^{18}$ Así, Habermas concibe a este principio como un uso específico del lenguaje que presupone exigentes compromisos asumidos por el hablante. De hecho, el sujeto que plantea pretensiones de validez a través

\footnotetext{
16 Aunque este trabajo fue publicado por primera vez en ese año, aquí se tendrá en cuenta la cuarta edición en alemán publicada por Suhrkamp en 1994, que el autor presenta con una bibliografía revisada y ampliada.

${ }^{17}$ Habermas, J., Faktizität und Geltung, p. 138.

18 Cfr. Maliandi, R.; Ética: dilemas y convergencias, pp. 231-240; Damiani, A.; "Acción y racionalidad en la filosofía trascendental: del giro copernicano al giro lingüísticopragmático", en Michelini, D., Soto, H., De Miguel, J. (eds.), Ética del discurso. Desafíos de la interculturalidad y la religión en un mundo global, Río Cuarto, ICALA, 2011, pp. 31-39.
} 
del discurso presupone también implícitamente que: 1) todos los interlocutores pueden participar en dicho proceso, 2) todos los participantes pueden expresar su opinión, presentar nuevos argumentos, criticar el argumento en discusión, y 3) nadie puede ser privado, por medio de coacción ejercida dentro o fuera del discurso, del ejercicio de los derechos establecidos en 1 o $2 .{ }^{19}$

Así, estos presupuestos del discurso argumentativo, implícitamente aceptados y formalmente reconocidos en todo orden institucional de un estado democrático de derecho, constituyen normas procedimentales sin las cuales un régimen no sería considerado democrático, porque justifican el valor epistémico y la validez normativa de las decisiones adoptadas en virtud de esas normas.

Estrechamente relacionado con el principio del discurso se encuentra el principio democrático de esta teoría política. Este principio, que al igual que el (principio) anterior, no reconoce las decisiones de la mayoría como decisiones concluyentes, confiere legitimidad y validez a las leyes y decisiones vigentes si fueron resultado de un procedimiento basado en relaciones simétricas de reconocimiento intersubjetivo, en el que diferentes tipos de razones pueden ( $\mathrm{y}$ tienen el derecho a) ser expuestas ${ }^{20}$.

Ahora bien, esta es sólo una primera aproximación (y presentación) general de la democracia deliberativa. Para dar una respuesta conceptualmente sólida al tema de este trabajo, a continuación corresponde señalar primero (y brevemente) alguno de los rasgos distintivos de la filosofía habermasiana, como es el sentido reconstructivo de la misma, para luego comenzar a explicitar una parte de los fundamentos filosóficos de esta teoría política, relacionada con la pragmática universal del lenguaje, y cuya tematización de las pretensiones

${ }^{19}$ Cfr. Habermas, J., Moralbewusstsein und kommunikatives Handeln, Frankfurt, Suhrkamp, 1983, p. 103; Faktizität und Geltung, pp. 155-157; “¿Qué significa pragmática universal?” (1976), en Habermas, J., Teoría de la acción comunicativa. Complementos y estudios previos, Madrid, Cátedra, 1997, p. 140, y Wahrheit und Rechtfertigung, Frankfurt, Suhrkamp, 1999, p. 244.

${ }^{20}$ Cfr. Habermas, J., Faktizität und Geltung, pp. 139, 141-142; Wabrheit und Rechtfertigung, p. 253; Nino, C. S., La constitución de la democracia deliberativa, Barcelona, Gedisa, 2003, pp. 166-167. 
universales de validez del discurso argumentativo permitirá luego justificar en este marco teórico la relación entre política y moral.

\section{Lenguaje y sentido reconstructivo de la filosofía habermasina}

El carácter reconstructivo de la filosofía habermasiana se expresa en sus teorías del derecho, de la política y de la ética. Esta tarea de reconstrucción racional puede leerse a la luz de la distinción de Ryle entre know how y know that, es decir, entre el saber cómo y el saber qué. ${ }^{21}$ Mediante esta distinción se diferencia entre la capacidad de un sujeto para formar oraciones y comunicarse con otros, por un lado, y las reglas y presupuestos que lo hacen posible, por el otro. En una tal labor reconstructiva el intérprete intenta así explicitar las reglas que posibilitan la producción simbólica; se trata de un análisis realizado desde un metanivel pero que a la vez indaga en la estructura profunda/interna del lenguaje y la comunicación, y ello a fin de clarificar las condiciones generales de todo entendimiento posible. Habermas señala aquella analogía entre la diferenciación del know how y el know that y las reconstrucciones racionales en los siguientes términos:

Podemos distinguir entre know how, o la capacidad de un sujeto competente que sabe producir o efectuar una determinada operación, por un lado, y el know that, es decir, el saber explícito acerca de cómo hace el sujeto para entender esa cosa, por otro (...). El hablante, en la medida en que su expresión [lingüística] es una expresión bien formada, y, por lo tanto, inteligible, la produce conforme a determinadas reglas y en virtud de determinadas estructuras. Domina el sistema de reglas de su lengua y sabe cómo aplicarlo en cada contexto, tiene de ese sistema de reglas un saber preteórico (...). Esta conciencia implícita de reglas es un know how. El

${ }^{21}$ Cfr. Ryle, G., The Concept of Mind, Londres, New York, Hutchinson's University Library, 1949. Es importante aclarar que este método reconstructivo habermasiano en parte se vale de las investigaciones desarrolladas por las ciencias empíricas aplicadas en campos como la lingüística o la psicología, entre los que se encuentran los estudios de Chomsky y Piaget que el mismo Habermas tiene en cuenta. Cfr. Chomsky, N., Aspects of Theory of Syntax, Cambridge, Cambridge University Press, 1965; Piaget, J., Introduction à l'épistémologie génétique, París, PUF, 1950. 
intérprete, a su vez, que no solamente comparte este saber implícito del hablante competente, sino que quiere entenderlo, tiene que transformar el know how en un saber explícito, es decir, en un know that de segundo nivel. Esta es la tarea de la comprensión reconstructiva, es decir, de la explicación de significados en el sentido de una reconstrucción racional de las estructuras que subyacen a la producción de formas simbólicas. ${ }^{22}$

La idea es que los sujetos saben cómo interactuar comunicativamente, cómo formar oraciones inteligibles, pero sin que esto implique necesariamente un saber acerca de las reglas que posibilitan este uso del lenguaje; uno puede producir enunciados con sentido, argumentos correctos, etc., sin saber que para esto está realizando ciertas operaciones, aplicando ciertos criterios, y siguiendo determinadas reglas. ${ }^{23}$ En este punto es posible entrever un cierto paralelismo con el análisis conceptual llevado a cabo por una parte de la tradición analítica de la filosofía contemporánea, pues se trata de un tipo de análisis que podría entenderse como un trabajo intelectual consistente en desmenuzar ideas o conceptos, en el sentido de descubrir cuáles son los componentes cognoscitivos - por cierto, no explícitamente reconocidos- de quienes hacen uso del lenguaje, y que subyacen a la práctica del mismo (por esto se habla de un saber preteórico). Éstos en un sentido ciertamente conocen las reglas, pero porque las usan (know how), pero en otro las ignoran en la medida en que no son capaces de explicitar cuáles son (know that). El ejemplo de esto sería la gramática, cuyo dominio práctico no lleva consigo la habilidad de enunciar sistemáticamente las reglas que la rigen. ${ }^{24}$

De lo que se trata entonces es de reconstruir (en términos de una pragmática universal) las presuposiciones "universales y necesarias" de los procesos de entendimiento posible; esto no implica una mera interpretación de expresiones simbólicas particulares, sino la explicitación de un saber o capacidad de tipo universal. El procedimiento reconstructivo no opera entonces sobre la estructura superficial de una lengua (o cultura, o forma de vida, etc.), sino que tiene por objeto revelar el sistema de reglas subyacentes que posibilitan la

\footnotetext{
${ }^{22}$ Habermas, J. “Qué significa 'pragmática universal'?”, p. 311.

${ }^{23}$ Cfr. McCarthy, T.; La teoría crítica de Jürgen Habermas, Madrid, Tecnos, 1987, p. 320.

${ }^{24}$ Cfr. Strawson, P.; Análisis y metafísica, Barcelona, Paidós, 1992, p. 41 y ss.
} 
producción de configuraciones simbólicas provistas de sentido entre interlocutores que se relacionan mediante un uso comunicativo del lenguaje. ${ }^{25}$

Así, Habermas establece un procedimiento metódico que explicita el saber preteórico del que los hablantes se valen en la interacción comunicativa cotidiana orientada al entendimiento intersubjetivo. Esto revela que la labor reconstructiva de las presuposiciones del lenguaje se lleva a cabo teniendo en cuenta, entonces, la dimensión pragmática del mismo.

\section{La relación política-moral en la democracia deliberativa}

A fin de hacer explícita la relación que en efecto se establece entre estas dos partes de la filosofía práctica en el marco de la política deliberativa, primero corresponde centrar la atención en el análisis de la dimensión pragmática del lenguaje, desarrollado por Habermas desde la década de 1970. Se trata entonces, en primer lugar, de identificar los presupuestos de la acción consistente en plantear un discurso argumentativo, y ello a fin de mostrar luego, en segundo lugar, cómo esto se expresa también en el marco conceptual de la teoría deliberativa de la democracia.

\section{4.a. Pragmática universal del lenguaje. Un análisis reconstructivo de las pretensiones de validez del discurso argumentativo}

Desde C. Morris, cuando se habla del componente pragmático del significado, a diferencia del componente sintáctico (que estudia la relación de los signos con otros signos), o semántico (que analiza las relaciones de los signos con la realidad), se hace mención al análisis de las relaciones de los signos con sus usuarios, con su empleo y con sus efectos ${ }^{26}$. En lo que respecta a la

${ }^{25}$ Cfr. Habermas, J. “Qué significa 'pragmática universal'?”, p. 313 y ss.; McCarthy, T.; La teoría crítica de Jürgen Habermas, p. 320 y ss.

${ }^{26}$ Cfr. Morris, C., Foundations of the Theory of Signs, Chicago, Chicago University Press, 1938. En realidad, esta nueva concepción de la capacidad significativa del lenguaje se evidencia también en los estudios que al respecto propuso ya en 1934 el filósofo alemán K. Bühler. En efecto, este autor identificó las funciones del lenguaje situando la expresión lingüística en su triple relación con el mundo, el hablante y el oyente. Así, 
concepción habermasiana de la pragmática del lenguaje, ésta se basa en la idea de que no sólo los rasgos fonéticos, sintácticos y semánticos de las oraciones, sino también, y de éstos se ocupa, ciertos rasgos pragmáticos (en el sentido de la competencia comunicativa) admiten una reconstrucción racional en términos universales. Así, el procedimiento reconstructivo de la pragmática universal de Habermas explicita las pretensiones de validez que todo hablante competente plantea con la expresión de cualquier acto de habla con sentido, y suponen una determinada capacidad por parte del mismo que le permite desenvolverse en ámbitos diversos y ante interlocutores diferentes.

En su escrito de 1976 (“¿Qué significa pragmática universal?”) afirma ya el filósofo que la expresión o manifestación de un enunciado satisface tres tipos de pretensiones de validez, estas tienen que ver con que los oyentes lo consideren verdadero en la medida en que refleje algo perteneciente al mundo, veraz en la medida en que exprese las intenciones del hablante, y normativamente correcto si afecta expectativas socialmente reconocidas. ${ }^{27}$ La pragmática universal del lenguaje analiza, más allá de la inteligibilidad que se exige a la dimensión gramatical de una oración, la capacidad de comunicación del hablante para hacerse entender por medio de determinados actos de habla que presuponen aquellas pretensiones de validez señaladas. En correspondencia con las mismas, dicha capacidad del hablante se manifiesta en su aptitud pragmática para formular oraciones referidas a diversos contextos comunicativos, eligiendo el contenido enunciativo que permita dar cuenta de una experiencia o un hecho (pretensión de verdad), manifestando sus intenciones de modo que la expresión lingüística exprese lo que verdaderamente piensa (pretensión de

observó que la función de exposición (Darstellung) o función expositiva, es sólo una de las tres funciones cooriginarias del lenguaje. En este sentido su tesis es que el lenguaje representa un medio que cumple a la vez tres funciones distintas, pero internamente asociadas entre sí. Las expresiones empleadas comunicativamente sirven para exponer estados de cosas, para dar expresión a las intenciones de un hablante, y para entablar relaciones con un destinatario (Bühler, K., Sprachtheorie. Die Darstellungsfunktion der Sprache, Jena, G. Fisher, 1934). Cfr. Habermas, J., Pensamiento postmetafísico, Madrid, Taurus, 1990, pp. 80 y 109-110. Para una lectura crítica de este autor, véase también Apel, K.-O., Semiótica filosófica, Buenos Aires, Almagesto, 1994, p. 273 y ss.

${ }^{27}$ Habermas, J., “¿Qué significa 'pragmática universal’?”, p. 299 y ss. 
veracidad), y ejecutando el acto de habla en cuestión cumpliendo con ciertas normas reconocidas (pretensión de rectitud normativa).

En estas pretensiones de validez que señala Habermas y con las que, respectivamente, las oraciones pueden emplearse en las funciones pragmáticas universales de exposición, de autopresentación, y de establecimiento de relaciones interpersonales, se expresa la competencia comunicativa para la que establece una investigación en términos de pragmática universal. ${ }^{28}$

El núcleo de la concepción habermasiana del lenguaje apunta entonces a la reconstrucción de los presupuestos inherentes a la racionalidad comunicativa, los cuales dan cuenta de la base de validez del habla en términos de sus correspondientes reglas constitutivas de hecho presupuestas por los interlocutores discursivos, puesto que las mismas posibilitan la formulación inteligible de enunciados y el consecuente entendimiento intersubjetivo. Habermas se propone así articular una concepción de la racionalidad sobre la base de una reconstrucción reflexiva de tales condiciones, adoptando para ello una concepción metodológica para el análisis de los actos de habla que pretende combinar el punto de vista universal y el socio-histórico como condición de validez de las decisiones adoptadas.

La pragmática universal del lenguaje, y su correspondiente diferenciación entre pretensiones universales de validez, constituye uno de los fundamentos filosóficos de la democracia deliberativa, puesto que permite expandir el concepto mismo de validez, que tradicionalmente se vinculaba exclusivamente con la verdad. En efecto, la aptitud para justificar las pretensiones de validez respecto de determinados temas mediante confrontación de argumentos es la nota definitoria de la idea misma de la racionalidad en cuanto racionalidad discursiva. En este sentido, el procedimiento intersubjetivo de fundamentación de decisiones y normas que establece la democracia deliberativa como condición de validez de las mismas exige que los interlocutores discursivos involucrados sólo se guíen, dice Habermas, por la "fuerza de coacción" que ejercen los mejores argumentos.

Ahora bien, y éste es el punto en cuestión que interesa resaltar respecto de la pragmática universal del lenguaje y su "aporte teórico" a nuestro tema, la

${ }^{28}$ Ibidem, pp. 328-329. 
mencionada explicitación reconstructiva de los presupuestos del discurso argumentativo permite identificar determinados principios de equidad discursiva que comportan un carácter moralmente distintivo, lo cual otorga sustento conceptual a la idea de De Zan de que en efecto es posible justificar la relación entre ética y política en el marco teórico de la democracia deliberativa.

\section{4.b. Reconstrucción de las pretensiones universales de validez y el lugar de la moral en la política deliberativa}

La democracia deliberativa de Habermas, a diferencia de la concepción de lo político de Hobbes o de Rousseau, pero también presente en autores contemporáneos como Rawls, no adopta un sentido contractualista (o constructivista), sino reconstructivo de los presupuestos de la interacción comunicativa. En efecto, uno de los fundamentos filosóficos antes señalado de esta teoría política que evidencia dicho sentido reconstructivo (también mencionado) es la pragmática universal del lenguaje, centrada en el análisis de los presupuestos normativos (de carácter pragmático) inherentes a las acciones que realizan los hablantes cuando hacen un uso argumentativo del lenguaje, orientado a intentar obtener una solución racional a un problema teórico o (en este caso) práctico.

El sentido reconstructivo de la pragmática universal del lenguaje analiza, entonces, la capacidad de los hablantes para comunicarse y ser entendidos por medio de ciertos actos de habla que presuponen estas pretensiones de validez, y situando el problema de la justificación de las mismas no sólo en el contexto de la verdad, sino también y fundamentalmente en el ámbito de la comunicación y del discurso. Así, la pragmática universal del lenguaje hace explícito lo que todo hablante competente (que plantea pretensiones de validez a través de un discurso argumentativo) tiene que presuponer y aceptar como condiciones normativas de posibilidad y de validez de la argumentación, y de la consecuente comprensión intersubjetiva. ${ }^{29}$

${ }^{29}$ Cfr. Habermas, J., “¿Qué significa 'pragmática universal’?”, pp. 328-329 y 299-300; Apel, K.-O., Semiótica filosófica, p. 315; Auseinandersetzungen in Erprobung des transzendentalpragmatischen Ansatzes, Frankfurt, Suhrkamp, 1999, p. 397; Böhler, D., 
Como resultado de esta explicitación es posible identificar la relación entre moral y política deliberativa que ahora es reconocida sólo implícitamente por Habermas, y ello en tanto que los referidos presupuestos expresan el valor moral que comporta el reconocimiento de los mismos. En este sentido, y aun cuando actualmente el filósofo parece concebir al principio del discurso en términos moralmente neutrales (porque a su entender el principio moral surge de "una posterior especificación del principio del discurso para las normas de acción" 30 ), si se tienen en cuenta sus consideraciones teóricas respecto de la ética del discurso, se evidencia la mencionada conexión entre política y moral que es posible fundamentar en el marco teórico de la democracia deliberativa, puesto que ellas también resultan inherentes al planteamiento conceptual de esta última en lo referente a su principio del discurso. En efecto, respecto de aquella teoría ética afirma el autor que las condiciones universales de validez de los juicios y normas morales se satisfacen en el contexto de un mundo de relaciones interpersonales bien ordenadas, las cuales se miden por las relaciones de reconocimiento reciproco que merecen ser aceptadas como justas por todos los participantes ${ }^{31}$. Cuando en el marco de la tematización de su teoría moral Habermas refiere a tal reconocimiento, está pensando en los presupuestos reconstructivamente explicitados por la pragmática universal del lenguaje y

Rekonstruktive Pragmatik. Von der Bewusstseinsphilosophie zur Kommunikationsreflexion: Neubegründung der praktischen Wissenschaften und Philosophie, Frankfurt, Suhrkamp, 1985, pp. 242 y 243. Aunque desde otra perspectiva filosófica, también R. Brandom refiere a la explicitación del conocimiento inherente al uso comunicativo del lenguaje (Brandom, R., Making it Explicit. Reasoning, Representing and Discursive Commitment, Cambridge, (Mass.), Harvard University Press, 1994, p. 77.

${ }^{30}$ Habermas, J., Faktizität und Geltung, p. 139. De hecho, fue a partir de esta afirmación que, para su colega Karl-Otto Apel, Habermas habría renunciado a los fundamentos filosóficos de la ética del discurso que juntos contribuyeron a crear en la década de 1970. En relación con la conexión entre política y moral en el marco de la democracia deliberativa, cabe destacar sin embargo qué distinta era la postura de Habermas expresada en sus conferencias sobre "Derecho y Moral", dictadas en el marco de las Tanner Lectures de 1986 (y publicadas en Faktizität und Geltung), cuando en efecto el filósofo aceptaba explícitamente dicha conexión (cfr. Habermas, Faktizität und Geltung, pp. $563,567,568$ y 597$)$.

31 Cfr. Habermas, J., Wabrheit und Rechtfertigung, pp. 284-285. 
aceptados por los interlocutores que participan del proceso decisorio. De este modo, el filósofo explicita los presupuestos normativos cuyo contenido moral resulta inherente a las relaciones simétricas de reconocimiento presupuestas ya en los procesos de fundamentación.

Ahora bien, y teniendo en cuenta ahora la teoría política habermasiana, cabe destacar que tales presupuestos normativos también tienen que considerarse y reconocerse en el marco conceptual de la democracia deliberativa, porque otorgan legitimidad democrática y también validez epistémica al procedimiento político de producción y fundamentación de normas en el estado democrático de derecho. En efecto, en este punto es necesario recordar que para el filósofo el concepto de discurso práctico de la democracia deliberativa, implica "la suave coacción que representan las inevitables presuposiciones de la argumentación, [las cuales] exigen de los participantes la adopción de las perspectivas de todos los demás y la atención igualitaria de los intereses de todos ellos". ${ }^{32} \mathrm{Y}$ tales presuposiciones se expresan en el (antes referido) principio de simetría (también reconocido por el principio del discurso), el cual presupone a su vez el principio de autonomía, de justicia, no exclusión, no violencia, igualdad, libertad, y respeto mutuo, entre otros, es decir, principios normativos que pueden también caracterizarse como "principios morales" siempre reconocidos cada vez que se plantea una pretensión de validez, independientemente del tema objeto de discusión. En este contexto resulta asimismo importante subrayar que para Habermas el (también ya mencionado) principio democrático, fundamental en su teoría política, surge de la correspondiente especificación del principio del discurso para las normas de acción que se presentan en forma de derecho, que pueden justificarse no sólo con ayuda de fundamentos pragmáticos, sino también éticopolíticos $y$ morales. ${ }^{33}$

32 Ibidem, p. 306. Siguiendo la interpretación de G. Lohmann respecto de la diferenciación habermasiana entre ética y moral, ya en este punto es posible justificar la conexión entre esta última y la teoría política, puesto que, en opinión del aquél, "la pregunta por la moral comporta una interrogación desde una perspectiva imparcial respecto de lo que resulta igualmente aceptable para todos" (cfr. Lohmann, G., "MoralDiskurse", en Brunkhorst, H., Kreide, R., Lafont, C. (comps.), Habermas Handbuch. München, J. B. Metzler, 2009, p. 82).

${ }^{33}$ Cfr. Habermas, J., Faktizität und Geltung, p. 139. 
La democracia deliberativa, acorde con el trasfondo hegeliano-marxista de la filosofía habermasiana, permite superar entonces la tensión entre las concepciones procedimentales y sustantivas de la teoría política, y ello en base a una tematización de lo político en el que ambas caracterizaciones del mismo ya no se presentan como extremos opuestos. Por esto es que, y así concebida, esta teoría política no se limita a descubrir cómo es la realidad, o cómo se toman efectivamente las decisiones (aunque esto es algo que, por supuesto, se tiene en cuenta). Sobre la base de su carácter reconstructivo, con el cual se explicitan las presuposiciones inherentes de la dimensión pragmática del discurso argumentativo, la democracia deliberativa descubre (o, de nuevo, identifica) los presupuestos normativos y a la vez también "morales" que tienen que considerarse como criterio de posibilidad y validez de tales decisiones políticas adoptadas en contextos democráticos.

De este modo entonces, y adoptando la postura apeliana de "pensar con Habermas contra Habermas", a partir de aquí podría comenzar a analizarse críticamente también el (ya aludido) actual posicionamiento habermasiano sobre el tema de la relación entre ética y política en el marco de la democracia deliberativa, y ello sin al mismo tiempo afectar el estatuto de la filosofía política (y de la democracia deliberativa) como disciplina autónoma en el marco de la filosofía práctica.

\section{Reflexiones finales}

Para justificar la tesis de su trabajo De Zan pone énfasis en las condiciones morales de los procesos decisorios, discursiva e intersubjetivamente mediados. En este marco alude a las presuposiciones del discurso argumentativo, pero sólo mencionando, y no tematizando, las correspondientes pretensiones universales de validez. Es cierto que hace una mención a este tema en una extensa nota, pero sin siquiera identificar tales pretensiones normativo-morales inherentes al planteo de todo discurso argumentativo; él mismo reconoce, en efecto, que "no desarrolla ni discute esta cuestión [que, sin embargo, es] fundamental"34. Esto reviste una importancia

${ }^{34}$ De Zan, J.; "La significación moral de la democracia", nota 23 de pp. 317-318. 
teórica esencial para el análisis del tema debido a que, precisamente, es a partir de la identificación y consecuente análisis de tales pretensiones de validez que corresponde fundamentar la tesis según la cual es posible explicitar la significación moral de la democracia. Por esta razón puede decirse, entonces, que el presente trabajo se propuso, más que realizar sólo una crítica al planteo de Zan, continuar su análisis del tema de la relación entre ética y política en el contexto de la democracia deliberativa, pero explicitando para ello las condiciones de posibilidad de tal relación a partir de reconstruir (parte de) los fundamentos filosóficos de esta teoría política.

Por supuesto que De Zan conocía (y obviamente explicitó) los presupuestos filosóficos de la teoría del discurso como fundamento de la teoría ética que en ella se basa (ética del discurso) y también de la democracia deliberativa. Prueba de esto son los numerosos trabajos publicados en formato de artículos y libros, y sus presentaciones como conferencista en diversos congresos, tanto de Argentina como del exterior. ${ }^{35}$ Ahora bien, en los últimos tiempos se volcó más hacia temas de filosofía política subrayando la autonomía de ésta respecto de la ética (lo cual de ningún modo significa que en su opinión no se pudieran relacionar). ${ }^{36}$ En todo caso, es el acto de retroceder a la pragmática universal del lenguaje, y a la consecuente explicitación reconstructiva de las reglas constitutivas de la dimensión pragmática del lenguaje argumentativo, lo que permite destacar aquellas condiciones normativas de validez que comportan un carácter moral. De este modo es

\footnotetext{
${ }^{35}$ Respecto de los análisis de De Zan sobre los presupuestos filosóficos de la teoría del discurso véase, entre otros trabajos, "Pragmática del lenguaje y racionalidad comunicativa", Stromata, XLVI, 1988, pp. 105-137; "La universalidad de las pretensiones de validez del habla", en Cuadernos del Sur, 25, 1992-1993; "Filosofía y pragmática del lenguaje", en Apel, K.-O., Semiótica filosófica, pp. 15-46. Respecto de sus trabajos sobre el planteamiento teórico de la ética del discurso, puede consultarse "La fundamentación de la ética en la época contemporánea", Tópicos, Santa Fe, 3, 1995, pp. 79-115; Panoramas de la ética continental contemporánea, Madrid, Akal, 2007; “¿Está agotado el programa de investigación de la ética del discurso?”, en Michelini, D., De Zan, J., Maliandi, R.; Ética del discurso. Recepción y críticas desde América latina, Río Cuarto, ICALA; "Conflicto de valores y de intereses. Dos límites de la ética del discurso", en Actas II, UCES, Buenos Aires, 2010, pp. 535-560.

${ }^{36}$ Cfr. De Zan, J., La vieja y la nueva política. Libertad, poder y discurso, Buenos Aires, UNSAM Edita, 2013, pp. 245-246, 248, 252-253.
} 
posible dotar del consecuente respaldo teórico a la tesis de la mencionada significación moral de la democracia, aquí analizada en el marco teórico de la política deliberativa.

Recibido: 03/2019; aceptado: 05/2019 